\title{
Preparation and characterization of optically-resonant atomically-flat nanosurface substrates for high-resolution scanning probe microscopy of single molecules
}

D.H. Dahayanaka, ${ }^{*}$ D.W. Kelle, ${ }^{*}$ D.J. Wasielewski, ${ }^{*}$ E.S. Day, ${ }^{*}$ D.R. White, ${ }^{*}$ T.D. Mishima, ${ }^{*}$ C.M. Waite, ${ }^{* *}$ J.L. Moore, ${ }^{* *}$ R.L. Halterman, ${ }^{* *}$ L.A. Bumm*

*Homer L. Dodge Department of Physics \& Astronomy, University of Oklahoma, 440 W. Brooks St., Norman, OK, 73019-2061

**Department of Chemistry \& Biochemistry, University of Oklahoma, 620 Parrington Oval, Norman, OK 73019-3051

The scanning tunneling microscopy (STM) has been broadly applied to measure electronic characteristics of individual molecules supported in an inert monolayer matrix, which is typically grown on gold thin films on mica (Figure 1) or bulk single crystal substrates [1]. Although these substrates are excellent for electronic measurements, they are not optically transparent which can complicate optical measurements. Flat gold nanoparticles (FGNPs) supported on indium tin oxide (ITO) coated glass (Figure 2a) can be used as optically-resonant atomically-flat substrates for STM studies. We discuss preparation and characterization of the FGNPs. Transmission electron microscopy (TEM) and scanning electron microscopy (SEM) shows that FGNPs can be prepared $100-5000 \mathrm{~nm}$ across with shapes that range from triangular to hexagonal with thicknesses of 15-25 $\mathrm{nm}$. In-focus multiple dark-field TEM imaging [2] confirms that the crystallographic orientation of the large facet is $\{111\}$ and the edges are in $<211>$ directions as seen in Figure $2 b$. Single-particle dark-field scattering spectroscopy and UV-vis-NIR spectrophotometry show the quadrupole plasmon resonance $\sim 800 \mathrm{~nm} \mathrm{[3]} \mathrm{and} \mathrm{the} \mathrm{dipole} \mathrm{plasmon} \mathrm{resonance} \mathrm{further} \mathrm{in} \mathrm{the} \mathrm{NIR,} \mathrm{which} \mathrm{are} \mathrm{size} \mathrm{and} \mathrm{the}$ shape dependent [4]. The addition of polyvinylpyrrolidone (PVP) as a stabilizer during growth effects the particle morphology, apparently slowing growth in the $<110>$ directions (Figure $3 \mathrm{a} \& \mathrm{~b}$ ). Also we demonstrate that single molecule electronic measurements can be performed using atomically-flat gold nanoparticles (FGNPs) supported on indium tin oxide coated glass as an alternative to traditional $\mathrm{Au}\{111\}$ substrates.

References

[1] (a) T.D. Dunbar et al., J. Phys. Chem. B, 104(20) (2000) 4880, (b) G.E. Poirier, Chem. Rev., 97 (1997) 1117.

[2] (a) J.W. Edington, Practical Electron Microscopy in Materials Science, Van Nostrand Reinhold Company, New York, 1976, p 13. (b) D.B. Williams and C.B. Carter, Transmission Electron Microscopy, Plenum Press, New York, 1996, p 188.

[3] E. Hao, G. C. Schatz, J. Chem. Phys., 120 (2004) 357.

[4] J.E. Millstone et al., J. Am. Chem. Soc., 127 (2005) 5312.

[5] A.I. Kirkland et al., Proc. R. Soc. London A, 440 (1993) 589.

[6] DHD thanks Oklahoma EPSCoR and CSPIN for a graduate research fellowship. We thank William Chisoe, III and Preston R. Larson for electron microscopy. This work was supported by the National Science Foundation CAREER grant No. CHE-0239803, the Center for Physics in Nanostructures, National Science Foundation MRSEC No. DMR-0080054, and Oklahoma EPSCoR. 


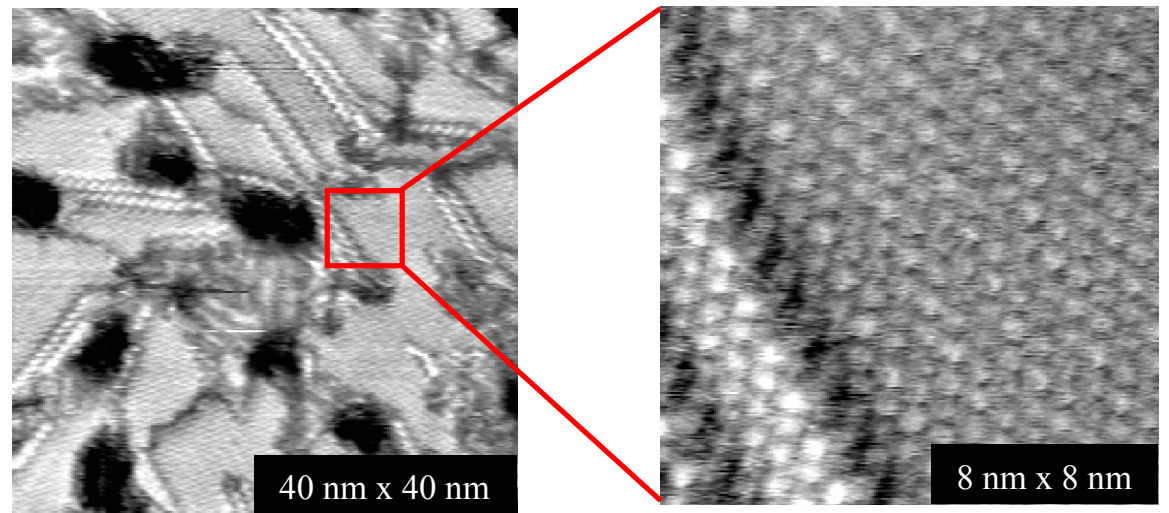

Figure 1. STM images of a decanethiol self assembled monolayer on Au/mica substrate. Tunneling conditions for both images are $-1 \mathrm{~V}_{\text {sample, }} 10 \mathrm{pA}$.
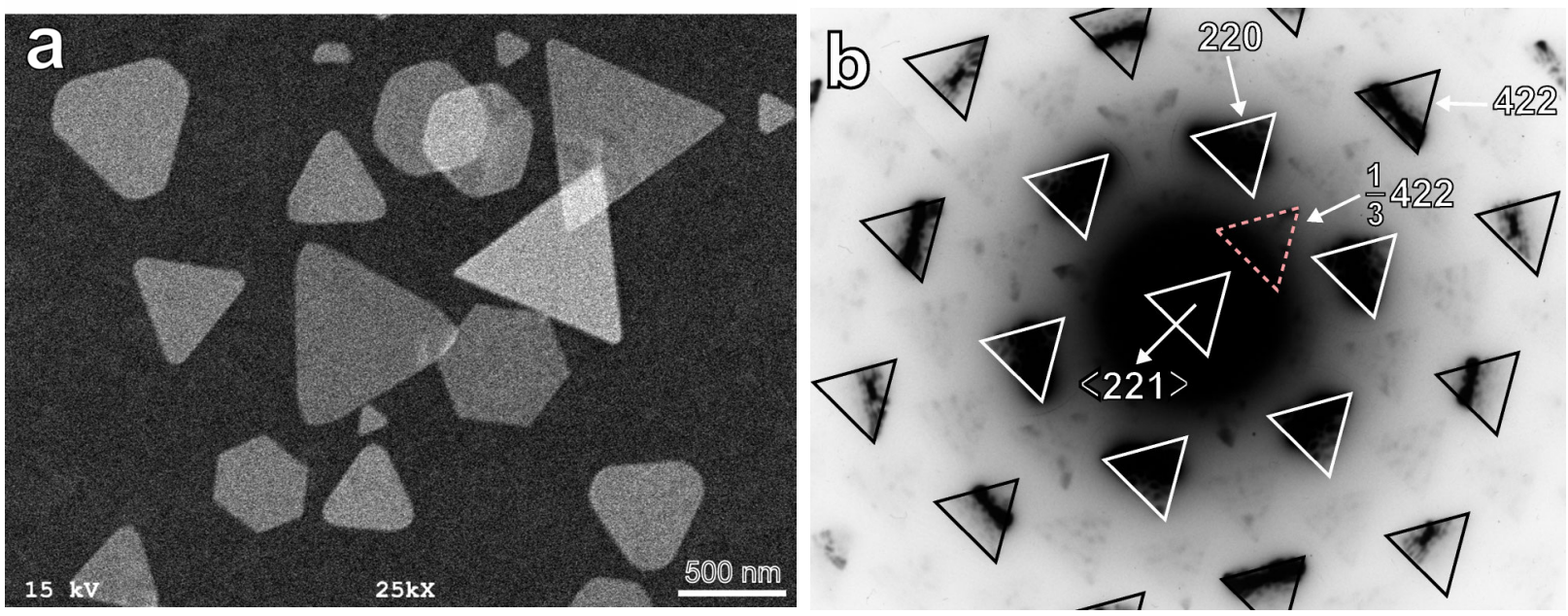

Figure 2. (a) SEM image of flat gold nanoparticles deposited on an ITO coated glass substrate. (b) In-focus multiple dark-field TEM images of a triangular shaped flat gold nanoparticle which shows the crystallographic orientation. The first ring of images, barely visible in the figure, are due to the normally forbidden 1/3 422 diffraction which appears in thin crystals [5].
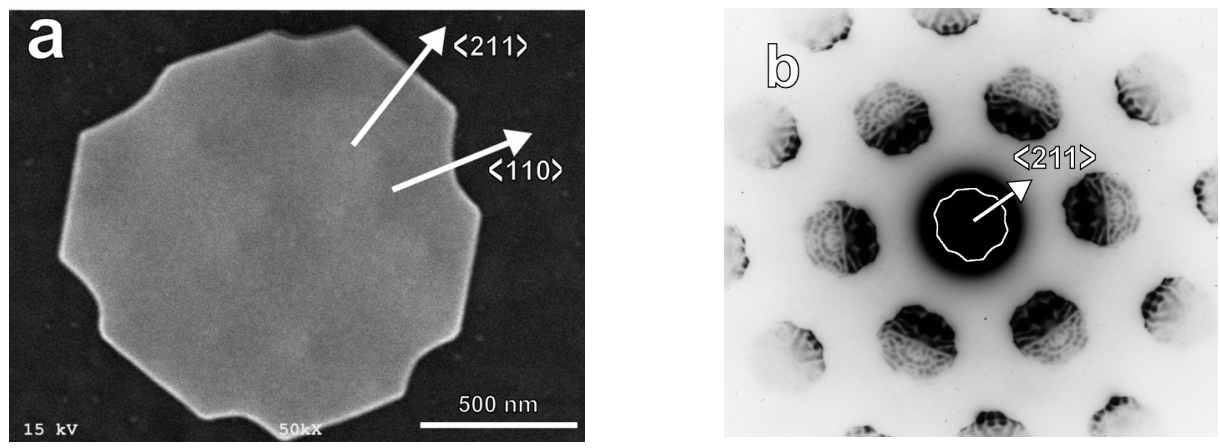

Figure 3. (a) SEM image of a PVP grown flat gold nanoparticle. (b) In-focus multiple dark-field TEM image of a PVP grown particle which shows the crystallographic orientations of the faces. 